\title{
Effects of horticultural therapy on elderly' health: protocol of a randomized controlled trial
}

\author{
Hui Yu Chan ${ }^{1}$, Roger Chun-Man Ho ${ }^{1,2^{*}}$, Rathi Mahendran ${ }^{1,2}$, Kheng Siang Ng${ }^{1}$, Wilson Wai-San Tam³ ${ }^{3}$ Iris Rawtaer ${ }^{1,2}$, \\ Chay Hoon Tan ${ }^{4}$, Anis Larbi ${ }^{5}$, Lei Feng ${ }^{1}$, Angelia Sia ${ }^{6}$, Maxel Kian-Wee $\mathrm{Ng}^{7}$, Goh Lee Gan ${ }^{8}$ and Ee Heok Kua ${ }^{1,2}$
}

\begin{abstract}
Background: Due to a rapidly ageing population in the world, it is increasingly pertinent to promote successful ageing strategies which are cost-effective, easily accessible, and more likely to be acceptable to the elderly. Past research associates exposure to natural environments and horticultural therapy (HT) with positive psychological, social and physical health benefits. This Randomized Controlled Trial (RCT) is designed to evaluate the efficacy of HT in promoting Asian elderly' mental health, cognitive functioning and physical health.

Methods/design: 70 elderly participants aged 60 to 85 years old will be randomized to participate in either the active horticultural therapy group or be in the waitlist control. Sessions will be weekly for 12 weeks, and monthly for 3 months. Mental health will be assessed through self-reports of depressive and anxiety symptomatology, life satisfaction, social connectedness and psychological well-being, collaborated with immunological markers. Outcome measures of cognitive functioning and physical health include neuropsychological tests of cognitive function and basic health screening. Outcomes will be assessed at baseline, 3 months and 6 months postintervention.

Discussion: This RCT comprehensively investigates the efficacy of a non-invasive intervention, $H T$, in enhancing mental health, cognitive functioning and physical health. The results have tremendous potential for supporting future successful ageing programs and applicability to larger populations.
\end{abstract}

Trial registration: ClinicalTrials.gov NCT02495194. Trial registration date: July 13, 2015. Retrospectively registered.

Keywords: Horticultural therapy, Gardening, Elderly, Mental health, Cognitive functioning, Physical health,

Randomized controlled trial

\section{Background}

Worldwide, one in every nine people is aged 60 years and above, and by 2050 the proportion is expected to increase to one in every 5 people [1]. In Singapore, there was a $2.2 \%$ increase in the proportion of older persons aged 65 years and above in the recent 5 years [2]. This population has greater mental health risks due to perceived social isolation [3]. Amongst the older adults, gardening is a popular leisure-time activity and exposure to

\footnotetext{
*Correspondence: roger_ho@nuhs.edu.sg

'Department of Psychological Medicine, Yong Loo Lin School of Medicine,

National University of Singapore, Singapore, Singapore

${ }^{2}$ Department of Psychological Medicine, National University Hospital,

Singapore, Singapore

Full list of author information is available at the end of the article
}

nature has been found to increase positive affect and reduction in anger in individuals, as opposed to those in the urban environment [4]. This may be due to the restorative effects of nature, such as recovery of directed attention fatigue and stress mitigation [5]. Therefore in recent years, there is burgeoning research on therapies which tap on the nature's benefits on human health and one of which is horticulture.

Definitions of horticultural activities in literature vary in scope from a broad perspective encompassing all forms of exposure to nature and plants, to a more narrow focus on active engagement with plants. Defined broadly, horticultural activities have been shown to reduce depression severity and rumination in clinically depressed patients as 
the garden environment offers a respite from the urban settings and fascination to them [6]. Notably, besides reducing negative mood (i.e. tension, depression, fatigue, anger and confusion), horticulture also brings about an increase in positive dimension (i.e. vigor/energy) [7]. Similarly, a 7-week indoor horticulture activity program was found to improve participants' psychological well-being, as compared to the waitlist control group [8]. Hence, horticultural activities may be a promising intervention in improving mental health.

An investigation on the efficacy of horticultural activities in improving mental health, cognitive functioning and physical health is merited for its wider implications. If proven to be effective, this innovative and costeffective intervention could be a nation-wide strategy in health promotion. It has been stated that the healthcare system alone may be limited in promoting public health [9] and that there is a need for public health strategies to focus on the socio-ecological aspects of health [10]. Therefore, urban parks, which are easily accessible for community-dwelling individuals, are an ideal resource to support the promotion of these aspects of well-being.

Because the present study is a goal-oriented intervention which seeks to enhance the mental, cognitive and physical health of the elderly participants, the definition put forth is adopted:

"Horticultural therapy (HT) is a professionally conducted client-centered treatment modality that utilizes horticulture activities to meet specific therapeutic or rehabilitative goals of its participants. The focus is to maximize social, cognitive, physical and/or psychological functioning and/or to enhance general health and wellness (p. 5)." [11].

Enhanced with the environmental benefits offered by the nature, HT may be a promising intervention in promoting public health and critical in prevention work. However, there is currently a paucity of research on the effectiveness of HT in a multi-ethnic Singaporean population as most studies were conducted either on western societies $[6,12,13]$ or Japan and Korea [14]. Due to unique social and cultural specificities, it is inappropriate to assume that the findings could be generalized to our local population.

Furthermore, there are limitations in the existing research. Most of the other studies either have small sample sizes [15-17], short intervention duration $[8,18]$, or employed the sole use of questionnaires without collaborating evidence from biological data. In addition, a systematic review found that the randomized controlled trials (RCT) conducted thus far have poor methodological and reporting quality [19]. Further research is therefore needed to determine the effectiveness of HT in improving the health of the elderly in the multi-ethnic Singaporean community.
Therefore the aims of this study are to investigate the effectiveness of HT in improving the mental, cognitive and physical health of the community-dwelling older adults using a RCT design. Based on the existing literature, it was hypothesized that, as compared to the waitlist control, participants in the active treatment group will 1) have better mental health, 3) have greater improvement of cognitive functioning and 3) have better physical health. In order to have collaborative evidence with measures of psychological health, the immunology of participants will be investigated via blood-based biomarkers. Notably, past research has found that depressive symptomatology was associated with elevated levels of pro-inflammatory cytokines [20] and lower levels of trophic factor [21].

\section{Methods}

All procedures involved in this trial will be conducted in compliance with the Helsinki declaration and Singapore Guidelines for Good Clinical Practice. The National University of Singapore Institutional Review Board (NUS IRB-Reference Code: B-15-016) gave ethical approval. This paper describes, according to SPIRIT guidelines, the trial design, setting, intervention and procedures that will be undertaken.

\section{Trial design}

This interventional study will be a RCT comparing participation in a HT program to being in a waitlist control. The intervention will lasts for 6 months with 1-h HT sessions during the first 3 months followed by monthly sessions the next 3 months. Outcome assessments will be conducted at baseline, 3 months and 6 months. The 3-months follow-up intervals were decided based on a study by Gonzalez et al. which found that participants' depressive symptoms declined after 3 months and these improvements were maintained in the final 6-month follow-up [6].

\section{Study participants}

Seventy elderly will be recruited from a research center in the western district of Singapore. All interested and potentially eligible participants will be screened for cognitive status and other eligibility criteria.

English and/or Mandarin-speaking community-dwelling participants aged between 60 to 85 years and have a minimum score of 22 and above on the Montreal Cognitive Assessment (MoCA) will be enrolled into the study. The cut-off score of 22 on MoCA is to select participants who have intact cognitive impairment [22]. However, older adults will be excluded from the study if they have 1) history of severe psychiatric conditions e.g. schizophrenia, bipolar disorder; 2) dementia; 3) significant visual or hearing impairment; 4) marked upper and lower limb motor 
difficulties, which may affect their ability to participate in the study; 5) are currently suffering from or have history of severe medical conditions e.g. cancer, stroke, Parkinson disease; or 6) are participating in another therapy at the same time.

\section{Recruitment and baseline procedures}

The flow of participants from recruitment to end of study is shown in Fig. 1. Potential participants who may fulfil the inclusion criteria will be invited to participate in the study. They will be contacted either through an invitation phone call or when they are at research centre for activities. The study will be explained in detail to potential participants with the participant information sheet (English and Mandarin versions available) before their written informed consent is obtained. They will be screened by trained research staff using MoCA and demographic questionnaire. Participants who meet the entry criteria will be enrolled into the study.

Following successful screening, enrolled participants will proceed with the rest of the baseline assessments. The participants will be subsequently randomised into either the active treatment group or waitlist control.
Utilizing the Random Allocation Software version 2.0 [23], randomization will be stratified by gender by the study-coordinator.

\section{Intervention}

The HT intervention is delivered in 1-h sessions, weekly in the first 3 months, followed by monthly sessions for the next 3 months (see Table 1). The extension of monthly sessions for 3 months is to determine sustainability and longer-term changes. Conducted by a trained practitioner and volunteer facilitators, the intervention is designed to cultivate an interest in gardening and promote relaxation. The participants will be assigned into groups of 7 with a facilitator for enhanced personal engagement. The participants will be with the same group members and facilitator throughout the intervention in order to build rapport and social connectedness. The facilitators are volunteers with the National Parks Board, who were trained with some basic knowledge of horticultural skills such as plant propagation, plant identification, flower arranging, houseplant care, and horticulture crafts. The essential element of the HT program is to communicate to the participants that plants are living

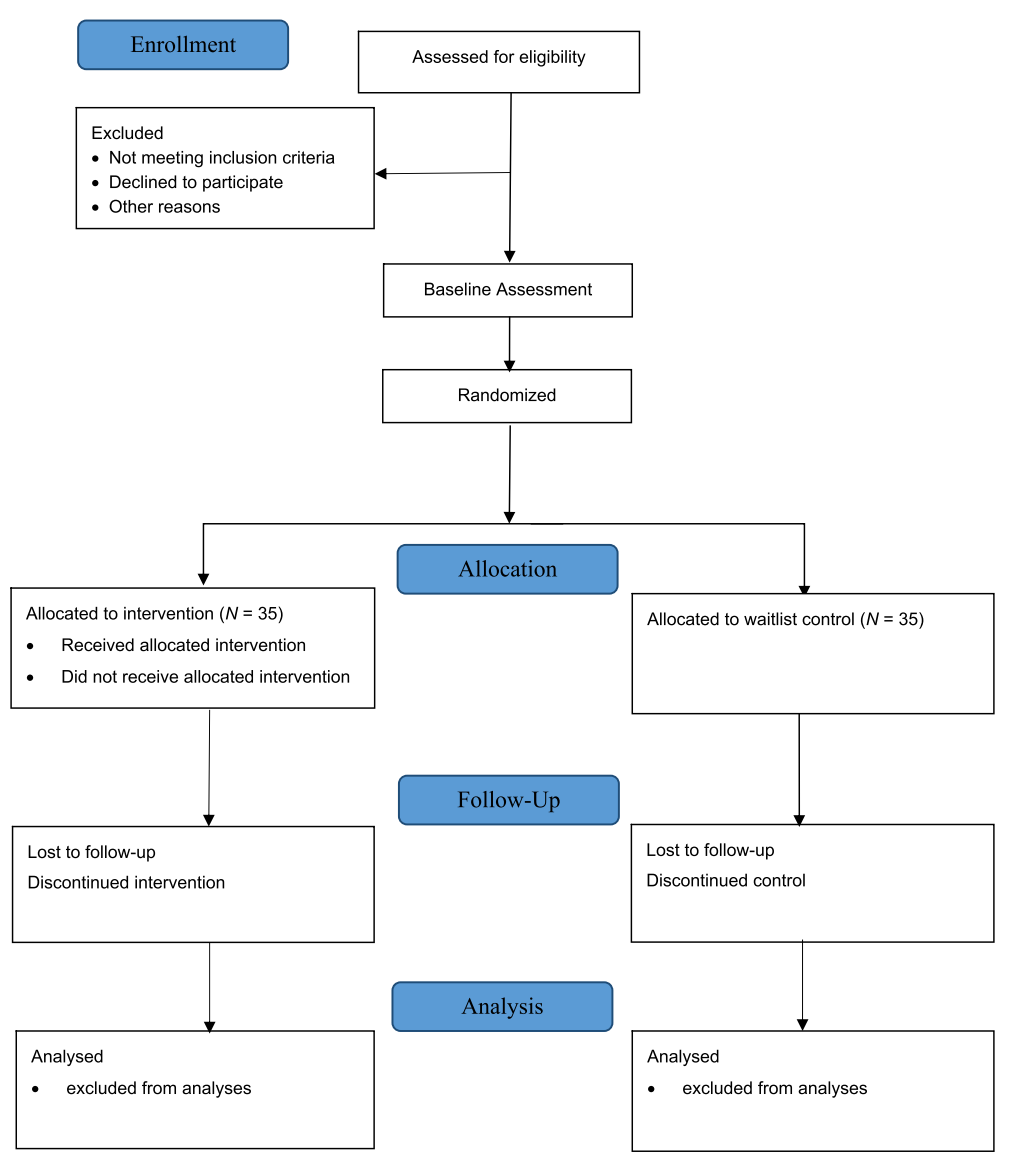

Fig. 1 Flow diagram of study protocol 
Table 1 Planned activity sessions for the 6 months of intervention

\begin{tabular}{|c|c|c|c|}
\hline Session & Topic & Activity & Venue \\
\hline 1 & Introduction & $\begin{array}{l}\text { 1. Familiarisation with research centre } \\
\text { 2. Group formation } 7 / \text { group } \\
\text { 3. Indoor Gardening basics }\end{array}$ & Research Centre \\
\hline 2 & Introduction & $\begin{array}{l}\text { 1. Garden familiarisation } \\
\text { 2. Vegetables growing brief } \\
\text { 3. Sowing vegetables seedlings }\end{array}$ & Chinese Garden \\
\hline 3 & Wetland Walk & $\begin{array}{l}\text { 1. Park amenities familiarization } \\
\text { 2. Interpretive walk } \\
\text { 3. Reflection }\end{array}$ & Sungei Buloh Reserve \\
\hline 4 & Introduction & $\begin{array}{l}\text { 1. Briefing for vegetables maintenance } \\
\text { 2. Weeding and fertilizing vegetables plot }\end{array}$ & Chinese Garden \\
\hline 5 & Nurturing & $\begin{array}{l}\text { 1. Briefing on pressed flowers } \\
\text { 2. Material preparation } \\
\text { 3. Make pressed flowers card }\end{array}$ & Research Centre \\
\hline 6 & Nurturing & $\begin{array}{l}\text { 1. Garden maintenance briefing } \\
\text { 2. Weeding, pruning, mulching garden/vegetables }\end{array}$ & Chinese Garden \\
\hline 7 & Colour Walk & $\begin{array}{l}\text { 1. Park amenities familiarization } \\
\text { 2. Interpretive walk } \\
\text { 3. Reflection }\end{array}$ & Singapore Botanical Gardens \\
\hline 8 & Nurturing & $\begin{array}{l}\text { 1. Vegetables maintenance } \\
\text { 2. Compost making brief } \\
\text { 3. Make compost }\end{array}$ & Chinese Garden \\
\hline 9 & Harvest and Cook & $\begin{array}{l}\text { 1. Harvest vegetables } \\
\text { 2. Hands on preparation for food } \\
\text { 3. Sharing of cooked vegetables }\end{array}$ & Research Centre \\
\hline 10 & Harvest and Cook & $\begin{array}{l}\text { 1. Seed sowing } \\
\text { 2. Herbal plant brief } \\
\text { 3. Herbal plants propagation }\end{array}$ & Chinese Garden \\
\hline 11 & Festive Walk & $\begin{array}{l}\text { 1. Park amenities familiarization } \\
\text { 2. Interpretive walk } \\
\text { 3. Reflection }\end{array}$ & Gardens by the Bay (Flower Dome) \\
\hline 12 & Harvest and Cook & $\begin{array}{l}\text { 1. Community Garden tour } \\
\text { 2. Plant care tips for herbs and plants } \\
\text { that will be brought home } \\
\text { 3. Reflection }\end{array}$ & Chinese Garden \\
\hline 13 & Healing Walk & $\begin{array}{l}\text { 1. Park amenities familiarization } \\
\text { 2. Interpretive walk } \\
\text { 3. Reflection }\end{array}$ & Botanical Garden - Healing Garden \\
\hline 14 & Gardening & $\begin{array}{l}\text { 1. Briefing } \\
\text { 2. Create a culinary garden } \\
\text { 3. Maintenance tips }\end{array}$ & Chinese garden - culinary garden creation \\
\hline 15 & Nature Walk & $\begin{array}{l}\text { 1. Park amenities familiarization } \\
\text { 2. Interpretive walk } \\
\text { 3. Reflection }\end{array}$ & Gardens by the Bay (Cloud Forest) \\
\hline
\end{tabular}

Intervention sessions 1 to 12 will be conducted on a weekly basis while sessions 13 to 15 will be conducted once a month

things that must be given care. This caring extends the emotional growth of the participants.

Each session will start with breathing and stretching exercises, before going into the activity (Table 1 ). The stretching exercises improve the flexibility of the participants, giving them more freedom of movement for the HT activities. In the first 3 months of weekly sessions, the intervention comprises of 3 main components: 1) indoor horticultural activities, 2) park visits and 3) outdoor gardening. The indoor activities will include making pressed flowers, which is an extension of appreciation for nature, indoor gardening to encourage participants to engage in gardening activity at their home and take ownership of their own plants.

Participants will be brought on visits to various urban parks in Singapore such as the Singapore Botanical Gardens, Sungei Buloh Wetland Reserve and Gardens by the Bay. These visit tours will be facilitated by trained park guides who will share their knowledge on the plants and the landscapes with the participants. Participants will also be encouraged to engage themselves actively in the environment by paying attention to the 
sights and sounds in the nature parks, thereby appreciating the nature and sharpening their focus. Viewing of natural spaces has been found to reduce anger and increase positive affect, due to the restorative effects of nature $[4,5]$.

For the gardening activities, they will be held fortnightly at the Chinese Garden where participants will be taught to do weeding and seed sowing. Such active participation in the natural environment has been found to improve mood state where participants were less stressed and were invigorated by their experiences [7]. Vegetables such as ladyfinger, milk cabbage, and choy sum will be planted by the participants. They will be educated on the making of fertilizer as well as knowledge on herbal plants. When their vegetable crops are ready for harvest, the participants will be able to prepare a soup dish together to encourage social connectedness amongst them.

Throughout the intervention programme, there will be an emphasis on education and mental stimulation whereby the facilitation is structured in terms of a series of questions and answers. This encourages active discussion amongst the facilitators and participants and social engagement. Longitudinal studies on mental stimulation and socially integrated lifestyle in late life have been found to have beneficial effect on cognition [24]. In closing each session, participants are asked for their reflection and verbal feedback.

The waitlist control will be assessed at the same timepoint as the active treatment group but will only receive the same intervention program 6 months later.

\section{Assessment}

Participants will undergo a thorough assessment using well-validated measures of mental health, biological samples analyses and physical health screening administered by trained research assistants and nurses. Most of the proposed measures have been employed in previous research and have good psychometric properties. These measures will have both English and Mandarin versions.

At baseline, socio-demographic data (such as age, gender, education and previous occupation), medical information, family history and lifestyle factors (such as gardening habits and frequency of park visits) will be obtained via a structured questionnaire. All baseline assessments will be completed in the 3 weeks before commencement of intervention.

\section{Measures of mental health}

Mental health is defined both in terms of psychopathology as well as positive well-being.

(1)Depressive and anxiety symptomatology of older adults will be measured by the Zung Self-Rating
Depression Scale (SDS) and the Zung Self-Rating Anxiety Scale (SAS).

(a) The SDS [25] is a 20-item quantitative measurement of symptoms of depression. Participants rate each item regarding how they felt during the week preceding using a 4-point scale that ranges from 1 (a little of the time) to 4 (most of the time). The higher the total score, the greater the severity of depressive symptoms. Several studies have established the SDS as a reliable and valid instrument for measuring depressive symptoms in Singaporean Chinese [26].

(b) The SAS [27] will be used to measure anxiety of the participants in the preceding week. It is a 20-item self-report assessment designed to measure anxiety levels, based on cognitive, autonomic, motor and central nervous system symptoms. Each question is scored on a Likert-type scale of 1-4 (a little of the time) to (most of the time). Some questions are negatively worded to avoid the problem of set response. Overall assessment is done by total score. The total raw scores range from 20 to 80 , with higher score indicating a greater severity of anxiety symptoms.

(2)Well-being will be assessed by the following instruments:

(a) The Ryff Scales of Psychological Well-Being [28] is an 18-item questionnaire which reflects the six areas of psychological well-being: autonomy, environmental mastery, personal growth, positive relations with others, purpose in life, and self-acceptance. Respondents rate statements on a scale of 1 to 6 , with 1 indicating strong disagreement and 6 indicating strong agreement.

(b)In measuring, life satisfaction, the Satisfaction with Life Scale (SWLS) will be administered [29]. SWLS is a 5-item scale designed to measure global cognitive judgments of one's life satisfaction (not a measure of either positive or negative affect). It is a psychometrically sound measure [30] and has been validated in a geriatric population [29]. Participants indicate how much they agree or disagree with each of the 5 items using a 7-point scale that ranges from 1 (strongly disagree) to 7 (strongly agree). Total scores range from 5 to 35 , with higher scores indicating higher level of life satisfaction. 
(c) The 6-item Friendship Scale [31] will be administered to measure both social isolation and social connectedness. Participants were asked to rate the frequency in which each statement describes them during the past four weeks on a 5-point Likert scale ranging from 0 (almost always) to 5 (not at all). Total scores range from 0 to 24, with higher scores indicating higher level of social connectedness. The scale was validated with older adults with excellent internal structures, reliability $(\alpha=0.83)$ and validity [31, 32].

(d)In order to gather biological evidence to collaborate with the self-report measures of psychological wellbeing, the immunology status of participants will be investigated via pro-inflammatory cytokines including Interleukin 6 (IL-6), Interleukin-1 beta (IL-1 $\beta$ ), C-reactive protein (CRP), Cortisol, Brain-derived neurotrophic factor (BDNF) and Dehydroepiandrosterone sulphate (DHEAS). Venipuncture will be carried out by qualified research nurses at the research centre. Venous blood $(10 \mathrm{ml})$ will be collected in cell preparation vacuum tubes with sodium citrate (BD plastic vacutainer \#362761) for collection of Plasma and Peripheral Blood Mononuclear Cells (PBMC). Samples will be delivered and processed within $3 \mathrm{~h}$ after the first venepuncture of the morning, which will be performed at room temperature. Once the samples arrive at the laboratory, 100ul of whole blood, for flow cytometry staining, will be aliquoted into Trucount tube. Subsequently, centrifugation of the samples will be performed at $1650 \mathrm{~g}$, for $25 \mathrm{~min}$ at room temperature.

After centrifugation, plasma will be aliquoted into several sterile $15 \mathrm{ml}$ tubes. Subsequently, PBMC will be re-suspended, transferred into a sterile $15 \mathrm{ml}$ tube and topped up with cold sterile Phosphate-buffered Saline (PBS) and 5\% Fetal Bovine Serum (FBS). PBMC will be centrifuged at $1500 \mathrm{rpm}$ for $5 \mathrm{~min}$ at $4^{\circ} \mathrm{C}$. 500,000 PBMCs will be aliquoted into $2 \mathrm{ml}$ Eppendorf tubes, processed and lysed in 600ul of miRVana Lysis buffer for downstream deoxyribonucleic acid/ ribonucleic acid (DNA/RNA) extraction. Remaining PBMCs will then be further processed, re-suspended in 90\% FBS and 10\% Dimethyl Sulfoxide (DMSO) and stored in cryovials. All samples will be stored at $-80^{\circ} \mathrm{C}$ until required. Biobanking will be in liquid nitrogen.

\section{Assessment of cognitive functioning}

(a) A battery of performance-based neurocognitive tests which evaluates across various cognitive domains including attention, learning, memory, visual-spatial skills and executive function will be administered by trained research assistants. This test battery has been adapted in local population with proved sensitivity in picking up differences with relatively small effect size [33, 34]. Clinical Dementia Rating (CDR) [35] is semi-structured, informant-based clinical interview which is rated based on a 5-point scale which characterize six domains of cognitive and functional performance applicable to Alzheimer disease and related dementias: memory, orientation, judgment \& problem solving, community affairs, home \& hobbies, and personal care. It has demonstrated convergent validity and discriminatory ability in the local population.

(b) The Montreal Cognitive Assessment (MoCA) [36] will be administered as global measures of cognitive function. MoCA is a brief screening tool for Mild Cognitive Impairment and Alzheimer Disease and was shown to have higher diagnostic accuracy and sensitivity to cognitive decline than the widely used Mini-Mental State Examination [22] and has been validated in the local population [37].

(c) Rey Auditory-Verbal Learning Test (RAVLT) [38] that evaluates immediate and delayed recall, learning rate, recognition, proactive and retroactive interference, primacy and recency will be administered

(d)Digit Span Task [39] that assesses attention and verbal working memory will be administered.

(e) To assess for sustained attention and sequencing, Colour Trails Tests [40] will be conducted.

(f) In measuring visual-spatial and organizational processing abilities, as well as non-verbal problemsolving skills, Block Design [39] will be administered

(g) Semantic Verbal Fluency (Animals) which taps on lexical knowledge and semantic memory organization will also be administered.

\section{Assessment of physical health}

A basic health screening of blood pressure, pulse rate, height and weight will be measured to investigate the effects of the therapy on physical health of the participants.

\section{Qualitative data}

Feedback questionnaire will be conducted to solicit participants' subjective experience and thereby expanding our understanding of the impact of the HT intervention. The survey will ask participants to identify what was the most helpful part of the class, what suggestions they have for improvement, and whether they had continued gardening and/or visiting the parks. This brief survey will only be administered once during the post-intervention assessment. Participants' responses will be informally tallied and listed in categories. 


\section{Sample size}

For a pilot RCT with continuous outcomes, the recommended total sample size is 70 , with 35 in each study arm with relative change in outcome variables within 95\% confidence interval [41].

\section{Data management}

Upon enrollment, each participant will be assigned a unique numeric study number so that they can be tracked anonymously throughout the study and only the Principal Investigator will have access to identifiers that can link the data to the individual participant. Consent forms and hardcopy data collection forms will be stored in a locked cabinet at the research centre and access to them is only made available through the Principal Investigator or trial coordinator.

All research data will be entered into electronic database. Two databases will be created: (1) administrative database which is a Microsoft Excel spreadsheet to compile data related to administrative matters and documentation process. They include identification data, administrative data and regulatory data and, (2) research database which includes a compilation of data that will be used for statistical analysis and outcome measures. This will encompass background data and research data. For this research database, data will be entered directly into Statistical Package for Social Sciences (SPSS) version 23.0 for ease of statistical analysis and the SPSS dataset will be duplicated onto Excel Spreadsheet to facilitate tracking of duplicate, missing and invalid values. For both databases, they will be secure and encrypted with password protection on an external hard drive. Access to the hard drive and password will only be available to Principal Investigator, delegated data manager and data entry staff.

To ensure the accuracy of the research data, three levels of data monitoring will be conducted whereby at the first level, the delegated data manager will check the hardcopies of the data collection forms to ensure accurate scoring. At the second level, data will be entered and at the third level, entries will be checked and approved by the delegated data manager.

\section{Biomarkers analyses and statistical analyses plan}

In the analyses of the biomarkers, aliquoted plasma will be used to measure the cytokines and metabolic profiles of the research participants using single-analyte enzymelinked immunosorbent assay (ELISA). Using targeted approach, selected RNA targets, which may include messenger RNA and micro-RNA, for a subset of the subject will be analysed based on the data obtained from the cytokines and metabolic profiles. Whole bloods aliquoted on Trucount tubes are stained with a series of fluorescence-conjugated antibodies marking the surface receptors of immune cell. Using flow cytometer, part of the phenotyping includes classical markers of senescence, Cluster of differentiation 57 (CD57) and Killercell lectin like receptor G1 (KLRG1), exhaustion of $\mathrm{T}$ cells, Programmed cell death protein 1 (PD1) and potentially identification of subpopulations of B cells and myeloid cells with a focus on the pro-inflammatory cells. The extent of DNA damage repair will be assessed by flow cytometry as well. Other immuno-assays aiming at identifying markers or predictors of immune status to examine the effects of the interventions may be applied with the bio-banked samples.

In the data analyses procedures, statistical significance level will be set at .05 for all descriptive and statistical analyses. All analyses will be performed using the SPSS. Based on the initial condition assignment and using the last observation carried forward (LOCF) method, intention-to-treat analyses will be conducted. Preliminary analyses will be performed next to assess the influence of demographic factors that (age, gender, education level, marital status, etc.). Where necessary, these variables will be included as covariates or control variables in the analyses. As some attrition is expected between interviews, prior to data analysis, comparisons will be made of retained and non-retained participants, to determine whether there are any systematic differences.

If there are chance imbalances in baseline participants' characteristics, they will be entered as covariates in the analyses. Repeated Measure Analysis of Variance (ANOVA) will be used to examine the difference of the outcome between the active treatment group and waitlist control group over the 3 time points [42]. If there are imbalances in baseline participants' characteristics, those variables will be included as covariates in the analyses. Additionally, dependent t-test will be employed to analyse the changes in scores within the active treatment group across the 3 time-points.

Ratios of selected biomarkers will be taken between cortisol, IL-6, CRP and DHEA-S. Additionally, fold changes and percentage changes would be performed. In the case of significant difference between the baseline measurements of prognostic demographic variables, multivariate regression will be performed when examining the differences between the means of the biomarkers for the two study arms. Correlation and/or heatmap analyses will be carried out to examine the relationships between the biomarker levels. On the aggregate biomarker level, principal component analyses (PCA) will be performed to examine the overall effect of the treatment. Mediational analyses employing bootstrapping technique will be employed to explore the mediating variables between the psychometric outcomes and biomarker levels. 


\section{Protocol compliance and deviation/violation}

A Note-to-file will be raised in the site investigator file and serious adverse events will be reported to the NUS-IRB.

\section{Discussion}

This RCT study has been designed to test the effectiveness of HT in improving the mental, cognitive and physical health of the older adults. Studies have shown that contact with nature has been associated with positive mood and improved sleep quality [13] and HT which allows for engagement with nature has been suggested to mitigate stress and improve mood state [7]. This study will be conducted on a multi-racial Asian population to provide substantiation if HT can improve mental health, cognitive functioning or physical health. With the objective measures of biomarkers as collaborative evidence, the proposed study will make significant contributions, both to a theoretical understanding of mental and cognitive health and to providing needed information to the healthcare providers coping with an ageing population.

In addition, this study is evaluating not only quantitative data but also qualitative data via feedback questionnaire. This will provide information on the subjective experience of the participants, and to inform areas of improvement for future programs as ultimately our focus will be on the benefits to the individual and the opportunities to continue such a program beyond the duration of the project.

HT may offer a playful and enjoyable experience to the participants, thereby providing an intrinsic motivation and increasing compliance. Hence, older adults would be selfmotivated to appreciate nature and be engaged in gardening even after the program ends. As a basic premise of this community-based initiative, we seek to build the capacity of the participants and empower them to take charge of their own health. This change in lifestyle will enhance their physical activity levels, and consequently increasing their health and ability to cope more effectively with the challenges faced in their daily life.

Community-based HT may provide an avenue for older individuals to socialize more and improve their mental health. After retirement, they are more likely to lead a sedentary lifestyle, with only $30 \%$ of them reaching the recommended level of leisure-time activity [43] and slightly more than half (58\%) in Singapore were sedentary [44]. Especially for those who are living alone, they might experience loneliness due to a lack of social interaction, which could in turn increase their risk for mental health problems [45]. Participation in HT will help build a sense of social connectedness amongst the older individuals. It is noteworthy that the proposed intervention also includes some physical exercise and educational components aiming to motivate the participants to carry on gardening at home. These various components aim to be holistic in delivering its benefits to the participants, as well as to encourage participants to adopt it as a lifestyle change. In addition, different locations will be used to conduct the intervention. Although this may add to the heterogeneity of such interventions, it reflects that such interventions, which are contingent on its environmental aspects, need to be adjusted to the local and cultural context.

The program can be easily translated to older adults' activities of daily living. Singapore, termed a "Garden City" has more than 350 parks which are close to homes for easy access [46]. Hence, urban parks are inexpensive resources readily available for the older adults to engage in walks and gardening activities. In addition, as part of the HT program, plants are given to participants to tend to at home, encouraging them to incorporate gardening as part of their daily routine.

Such community-based interventions supported by volunteers are important as they have the potential to be sustained. With its clinical implications, this initiative will guide future larger research trials and healthcare providers of the need to support cost-effective HT as a strategy to engage older adults in a more active and healthier lifestyle. In the context of the growing worldwide mental illness burden of disease, HT will go a long way in easing public healthcare, social and economic costs.

\section{Study progress}

The study has completed data collection.

Intervention programme for the waitlist control and data analyses are currently ongoing. Data analyses are estimated to be completed in October 2016.

\section{Abbreviations}

ANOVA: Analysis of Variance; BDNF: Brain-derived neurotrophic factor; CD57: Cluster of differentiation 57; CDR: Clinical Dementia Rating; CRP: Creactive protein; CRP: C-reactive protein; DHEAS: Dehydroepiandrosterone Sulphate; DMSO: Dimethyl Sulfoxide; DNA/RNA: Deoxyribonucleic acid/ Ribonucleic acid; ELISA: Enzyme-Linked Immunosorbent Assay; FBS: Fetal Bovine Serum; HT: Horticultural Therapy; IL-1B: Interleukin-1 beta; IL-

6: Interleukin 6; KLRG1: Killer-cell Lectin like Receptor G1; MoCA: Montreal Cognitive Assessment; PBMC: Plasma and Peripheral Blood Mononuclear Cells; PBS: Phosphate-buffered Saline; PCA: Principal Component Analyses; PD1: Programmed Cell Death Protein 1; RAVLT: Rey Auditory-Verbal Learning Test; RCT: Randomized Controlled Trial; SAS: Zung Self-Rating Anxiety Scale; SDS: Zung Self-Rating Depression Scale; SPSS: Statistical Package for Social Sciences: SWLS. Satisfaction with Life Scale

\section{Acknowledgements}

The investigators would also like to thank Ms. Toh Xiu Yong and the research nurses who assisted in data collection, the volunteers and various park guides who are supporting the horticultural therapy program as well as Ms. Crystal Tan Sze Ying for her support in biological markers.

Last but not least, the team would like to thank Lee Kim Tah Holdings Limited for their belief in our work and supporting the research activities at Jurong Point.

\section{Funding}

This research is funded by the National Parks Board and the Mind-Science Centre, National University of Singapore. Except for the intervention program National Parks Board had no role in the design of this study and will not have any role during its analyses, interpretation of the data, or decision to submit results. 


\section{Availability of data and materials}

Data are not made available because this paper is a protocol publication.

\section{Authors' contributions}

HYC assisted in the study design, coordinated the study procedures, carried out data collection and drafted the manuscript. RCH participated in the study design and provided guidance. KSN assisted in data collection and processing of blood samples. WWT provided statistical advice. RM, IR, CHT and GLG assisted in the study design and developed with other team members FL (neuropsychological tests) and AL (immunological markers). MKN and AS designed and supported the horticultural therapy program. EHK conceptualized the study, participated in the design of the study and gave intellectual inputs for the manuscript. All authors read and approved the final manuscript.

\section{Authors' information}

HYC: Research Assistant and Masters Candidate.

RCH: Associate Professor and Consultant Psychiatrist.

RM: Associate Professor and Senior Consultant Psychiatrist.

KSN: Doctor of Philosophy (PhD) candidate.

WWT: Assistant Professor.

IR: Associate Consultant Psychiatrist.

CHT: Associate Professor

LA: Principal Investigator Technologist.

LF: Research Assistant Professor.

AS: Assistant Director.

MKN: Senior Manager.

GLG: Professorial Fellow.

EHK: Senior Consultant Psychiatrist and Professor.

\section{Ethics approval and consent to participate}

This study obtained ethics approval from the National University of Singapore Institutional Review Board (NUS IRB-Reference Code: B-15-016). Informed consent will be obtained from all potential participants before trial commencement.

\section{Consent for publication}

Not applicable.

\section{Competing interests}

The authors declare that they have no competing interests.

\section{Publisher's Note}

Springer Nature remains neutral with regard to jurisdictional claims in published maps and institutional affiliations.

\section{Author details \\ 'Department of Psychological Medicine, Yong Loo Lin School of Medicine, National University of Singapore, Singapore, Singapore. ${ }^{2}$ Department of Psychological Medicine, National University Hospital, Singapore, Singapore. ${ }^{3}$ Alice Lee Centre for Nursing Studies, Yong Loo Lin School of. Medicine, National University of Singapore, Singapore, Singapore. ${ }^{4}$ Department of Pharmacology, Yong Loo Lin School of Medicine, National University of Singapore, Singapore, Singapore. ${ }^{5}$ Singapore Immunology Network, Agency for Science, Technology and Research, Singapore, Singapore. ${ }^{6}$ Centre for Urban Greenery and Ecology Research, National Parks Board, Singapore, Singapore. ${ }^{7}$ Horticulture \& Community Gardening Division, National Parks Board, Singapore, Singapore. ${ }^{8}$ Division of Family Medicine, National University Hospital, Singapore, Singapore.}

\section{Received: 2 March 2016 Accepted: 17 August 2017}

Published online: 29 August 2017

\section{References}

1. Guseh JS. Aging of the world's population. In: Shehan CL, editor. Wiley Blackwell Encycl. Fam. Stud. 1st ed. John Wiley \& Sons, Inc.; 2016. p. 1-5.

2. Singapore Department of Statistics. Population Trends 2015. Dep Stat Minist Trade Ind Singapore 2015.

3. Cornwell EY, Waite L. Social disconnectedness, perceived isolation, and health among older adults. J Health Soc Behav. 2009;50:31-48.
4. Hartig T, Evans GW, Jamner LD, Davis DS, Gärling T. Tracking restoration in natural and urban field settings. J Environ Psychol. 2003;23:109-23.

5. Kaplan S. The restorative benefits of nature: toward an integrative framework. J Environ Psychol. 1995;15:169-82.

6. Gonzalez MT, Hartig T, Patil GG, Martinsen EW, Kirkevold M. Therapeutic horticulture in clinical depression: a prospective study of active components. J Adv Nurs. 2010;66:2002-13.

7. Hayashi N, Wada T, Hirai H, Miyake T, Matsuura Y, Shimizu N, et al. The Effects of Horticultural Activity in a Community Garden on Mood Changes. Environ. Control Biol. 2008. p. 233-40.

8. Barnicle T, Midden KS. The effects of a horticulture activity program on the psychological well-being of older people in a long-term care facility. HortTechnology. 2003;13:81-5.

9. Hancock T. Health care reform and reform for health: creating a health system for communities in the 21st century. Futures. 1999;31:417-36.

10. Maller C, Townsend M, Pryor A, Brown P, St Leger L. Healthy nature healthy people: "contact with nature" as an upstream health promotion intervention for populations. Health Promot Int. 2006;21:45-54.

11. Haller R, Kramer C, editors. Horticultural therapy methods: making connections in health care, human service, and community programs. Binghamton, NY: The Haworth Press; 2006.

12. Derback IS, Derstr MS, Lander ES. Horticultural therapy: the "healing garden" and gardening in rehabilitation measures at Danderyd hospital rehabilitation clinic. Sweden Pediatr Rehabil. 2004;7:245-60.

13. Rappe E, Kivelä SL. Effects of garden visits on long-term care residents as related to depression. HortTechnology. 2005;15:298-303.

14. Matsuo E. Education, research, and use of human-horticulture relationships in Japan and Korea. HortTechnology. 2000;10:14-7.

15. Gonzalez MT, Hartig T, Patil GG, Martinsen EW, Kirkevold M. A prospective study of group cohesiveness in therapeutic horticulture for clinical depression. Int J Ment Health Nurs. 2011;20:119-29.

16. Kam MCY, Siu AMH. Evaluation of a horticultural activity programme for persons with psychiatric illness. Hong Kong J Occup Ther Elsevier. 2010;20:80-6.

17. Milligan C, Gatrell A, Bingley A. "cultivating health": therapeutic landscapes and older people in northern England. Soc Sci Med. 2004;58:1781-93.

18. Tse MMY. Therapeutic effects of an indoor gardening programme for older people living in nursing homes. J Clin Nurs. 2010;19:949-58.

19. Kamioka $\mathrm{H}$, Tsutani $\mathrm{K}$, Yamada M, Park H, Okuizumi H, Honda T, et al. Effectiveness of horticultural therapy: a systematic review of randomized controlled trials. Complement Ther Med Elsevier Ltd. 2014;22:930-43.

20. Baune BT, Smith E, Reppermund S, Air T, Samaras K, Lux O, et al. Inflammatory biomarkers predict depressive, but not anxiety symptoms during aging: the prospective Sydney memory and aging study. Psychoneuroendocrinology. 2012;37:1521-30.

21. Aydemir C, Yalcin ES, Aksaray S, Kisa C, Yildirim SG, Uzbay T, et al. Brain-derived neurotrophic factor (BDNF) changes in the serum of depressed women. Prog Neuro-Psychopharmacol Biol Psychiatry. 2006; 30:1256-60.

22. Freitas S, Simões MR, Alves L, Santana I. Montreal cognitive assessment: validation study for mild cognitive impairment and Alzheimer disease. Alzheimer Dis Assoc Disord. 2013;27:37-43.

23. Saghaei M. Random allocation software for parallel group randomized trials. BMC Med Res Methodol. 2004;4:26

24. Fratiglioni L, Paillard-Borg S, Winblad B. An active and socially integrated lifestyle in late life might protect against dementia. Lancet Neurol. 2004. p. 343-53.

25. Zung WWK. A self-rating depression scale. Arch Gen Psychiatry. 1965;12:63-70.

26. Chang WC, Koh JBK. A measure of depression in a modern asian community: Singapore. Depress Res Treat. 2012;2012

27. Zung WW. A rating instrument for anxiety disorders. Psychosomatics. 1971; 12:371-9.

28. Ryff CD, Keyes $C L$. The structure of psychological well-being revisited. J Pers Soc Psychol. 1995;69:719-27.

29. Diener E. Emmons R a., Larsen RJ, griffin S. The satisfaction with life scale. J Pers Assess. 1985:49:71-5.

30. Larsen RJ, Diener E, Emmons RA. An evaluation of subjective well-being measures. Soc Indic Res. 1985;17:1-17.

31. Hawthorne G. Measuring social isolation in older adults: development and initial validation of the friendship scale. Soc Indic Res. 2006;77:521-48.

32. Hawthorne G. Perceived social isolation in a community sample: its prevalence and correlates with aspects of peoples' lives. Soc Psychiatry Psychiatr Epidemiol. 2008:43:140-50. 
33. Feng L, Ng TP, Chuah L, Niti M, Kua EH. Homocysteine, folate, and vitamin B-12 and cognitive performance in older Chinese adults: findings from the Singapore longitudinal ageing study. Am J Clin Nutr. 2006;84:1506-12.

34. Feng L, Isaac V, Sim S, Ng TP, Krishnan KRR, Chee MWL. Associations between elevated homocysteine, cognitive impairment, and reduced white matter volume in healthy old adults. Am J Geriatr Psychiatry. 2013;21:164-72.

35. Morris JC. The clinical dementia rating (CDR): current version and scoring rules. Neurology. 1993;43:2412-4.

36. Nasreddine Z, Phillips N, Bédirian V, Charbonneau S, Whitehead V, Colllin I, et al. The Montreal cognitive assessment, MoCA: a brief screening tool for mild cognitive impairment. J Am Geriatr Soc. 2005:53:695-9.

37. Liew TM, Feng L, Gao Q, Ng TP, Yap P. Diagnostic utility of montreal cognitive assessment in the fifth edition of diagnostic and statistical manual of mental disorders: major and mild neurocognitive disorders. J Am Med Dir Assoc. 2015;16:144-8

38. Rey A. L'examen psychologique dans les cas d'encéphalopathie traumatique. Arch Psychol (Geneve). 1941;28:215-85.

39. Wechsler D. Wechsler adult intelligence scale-third edition, administration and scoring manual. Third. San Antonio, TX: The Psychological Corporation; 1997.

40. D'Elia L, Satz P, Uchiyama C, White T. Color trails test: professional manual. Psychological Assessment Resources: Odessa, FL; 1996.

41. Teare D, Hayman A, Dimairo M, Shephard N, Whitehead A, Walters S. Sample size requirements for pilot randomised controlled trials with continuous outcomes: a simulation study. Trials. 2013;14:P46.

42. Plichta SB, Kelvin EA, Munro BH. Munro's statistical methods for health care research. 6th ed. Philadelphia: Wolters Kluwer Health/Lippincott Williams \& Wilkins; 2013.

43. Ashe M, Miller W, Eng J, Noreau L. Older adults, chronic disease, and leisuretime physical activity. Gerontology. 2009;55:64-72.

44. Lian WM, Gan GL, Pin CH, Wee S, Ye HC. Correlates of leisure-time physical activity in an elderly population in Singapore. Am J Public Health. 1999;89:1578-80.

45. Coyle CE, Dugan E. Social isolation, loneliness and health among older adults. J Aging Health. 2012;24:1346-63.

46. Ministry of National Development. A city in a garden - more parks and park connectors [Internet]. 2013 [cited. Jul 20. 2016; Available from: http://www. mnd.gov.sg/LandUsePlan/city_in_a_garden.htm

\section{Submit your next manuscript to BioMed Central and we will help you at every step:}

- We accept pre-submission inquiries

- Our selector tool helps you to find the most relevant journal

- We provide round the clock customer support

- Convenient online submission

- Thorough peer review

- Inclusion in PubMed and all major indexing services

- Maximum visibility for your research

Submit your manuscript at www.biomedcentral.com/submit

) Biomed Central 